\title{
Heat and Mass Transfer on Squeezing Unsteady MHD Nanofluid Flow between Parallel Plates with Slip Velocity Effect
}

\author{
Khilap Singh, Sawan K. Rawat, and Manoj Kumar \\ Department of Mathematics, Statistics and Computer Science, G. B. Pant University of Agriculture and Technology, \\ Pantnagar, Uttarakhand, India \\ Correspondence should be addressed to Khilap Singh; singh.khilap8@gmail.com
}

Received 30 June 2016; Revised 17 October 2016; Accepted 24 October 2016

Academic Editor: In Cheol Bang

Copyright (C) 2016 Khilap Singh et al. This is an open access article distributed under the Creative Commons Attribution License, which permits unrestricted use, distribution, and reproduction in any medium, provided the original work is properly cited.

Heat and mass transfer behavior of unsteady flow of squeezing nanofluids between two parallel plates in the sight of uniform magnetic field with slip velocity effect is investigated. The governing equations representing fluid flow have been transformed into nonlinear ordinary differential equations using similarity transformation. The equations thus obtained have been solved numerically using Runge-Kutta-Fehlberg method with shooting technique. Effects on the behavior of velocity, temperature, and concentration for various values of relevant parameters are illustrated graphically. The skin-friction coefficient and heat and mass transfer rate are also tabulated for various governing parameters. The results indicate that, for nanofluid flow, the rates of heat and mass transfer are inversely proportional to nanoparticle volume fraction and magnetic parameter. The rate of mass transfer increases with increasing values of Schmidt number and squeeze number.

\section{Introduction}

The study of heat and mass transfer in unsteady squeezing viscous nanofluid flow between two parallel plates is a stimulating topic of exploration because of its industrial use and intense biological situations, some of which include processing of polymer, compression, power transmitting, lubricant system, transient loading of mechanical components and the squeezed films in power transmission, food processing, and cooling water, modeling of synthetics transportation inside living bodies, hydromechanical machinery, chemical processing equipment, and crop destruction due to freezing. The first work on the squeezing flow under lubrication approximation was studied by Stefan [1]. The flow analysis between two parallel plates of $\mathrm{Cu}$-water squeezing nanofluid was investigated by Domairry and Hatami [2]. Pourmehran et al. [3] studied the unsteady flow of squeezing nanofluid between parallel plates. Gupta and Ray [4] proposed a problem of unsteady flow of a squeezing nanofluid between two parallel plates. The squeezing flow of $\mathrm{Cu}$-water (or kerosene) nanofluid between two parallel plates under the effects of viscous dissipation and velocity slip was investigated by Khan et al. [5]. Dib et al. [6] obtained an approximate analytic solution of squeezing unsteady nanofluid flow.

The word nanofluid represents the fluid in which particles of size with order of nanometer (diameter $<100 \mathrm{~nm}$ ) are mixed in the base fluid. The nanoparticles used in nanofluids are generally made of metals $(\mathrm{Al}, \mathrm{Cu})$, oxides $\left(\mathrm{Al}_{2} \mathrm{O}_{3}, \mathrm{CuO}\right.$, $\mathrm{TiO}_{2}$, and $\mathrm{SiO}_{2}$ ), carbides $(\mathrm{SiC})$, nitrides (AlN, $\left.\mathrm{SiN}\right)$, and nonmetal (graphite, carbon nanotubes) and the base fluid is usually a conductive fluid, such as water or ethylene glycol. Other base fluids are toluene, oil, other lubricants, biofluids, and polymer solution. Nanoparticles are present up to 5\% volume fraction in nanofluids. The conventional heat transfer fluids are poor conductors of heat. Nanofluids make an edge over them because they have high heat transfer capability. Since these heating/cooling fluids play a vital role in the development of energy efficient heat transfer equipment for energy supply, to raise the thermal conductivity of these fluids, nanosized conducting metal particles are added to them. Therefore, their proper understanding is a must to use them efficiently in modern industry. Applications of nanofluids include microelectronics, fuel cells, and pharmaceutical processes. Choi and Eastman [7] were the first to propose the 
TABLE 1: Thermophysical properties of pure water and nanoparticles.

\begin{tabular}{lcccc}
\hline & $\rho\left(\mathrm{Kg} / \mathrm{m}^{3}\right)$ & $C_{p}(\mathrm{~J} / \mathrm{kgK})$ & $k(\mathrm{~W} / \mathrm{mK})$ & $\beta \times 10^{5}\left(\mathrm{~K}^{-1}\right)$ \\
\hline Pure water & 997.1 & 4179 & 0.613 & 21 \\
Copper $(\mathrm{Cu})$ & 8933 & 385 & 401 & 1.67 \\
Silver $(\mathrm{Ag})$ & 10,500 & 235 & 429 & 1.89 \\
Alumina $\left(\mathrm{Al}_{2} \mathrm{O}_{3}\right)$ & 3970 & 765 & 40 & 0.85 \\
Titanium oxide $\left(\mathrm{TiO}_{2}\right)$ & 4250 & 686.2 & 8.9538 & 0.9 \\
\hline
\end{tabular}

term nanofluid that represents the fluid in which nanoscale particles are suspended in the base fluid with low thermal conductivity such as water, ethylene glycol, and oil. In recent years, many researchers have studied and reported nanofluid technology experimentally or numerically in the presence of heat transfer [8-23].

Ibrahim and Shankar [24] studied MHD nanofluid flow and heat transfer over a stretching sheet in the presence of thermal radiation and slip conditions. Malvandi and Ganji [25] investigated effect of magnetic field on heat transfer of alumina/water nanofluid inside a circular microchannel. Ul Haq et al. [26] obtained the influence of thermal radiation and slip on MHD nanofluid flow passed over a stretching sheet. Govindaraju et al. [27] solved the problem of magnetohydrodynamic nanofluid flow on entropy generation in a stretching sheet with slip velocity. The problem based on effects of Stefan blowing and the slips on bioconvection nanofluid flow over a horizontal plate in motion was numerically investigated by Uddin et al. [28]. Hsiao [29] studied the MHD mixed-convection stagnation flow of a nanofluid over stretching sheet in the presence of slip. Kameswaran et al. [30] investigated chemical reaction and viscous dissipation effects on nanofluid flow through stretching or shrinking sheet. Matin and Pop [31] studied heat and mass transfer flow of a nanofluid with chemical reaction in porous channel. Pal and Mandal [32] observed mixed-convection heat and mass transfer stagnation-point flow in nanofluids through stretching/shrinking sheet in a porous medium with thermal radiation. The nanofluid flow and heat transfer in porous medium in the presence of magnetic field and radiation were made by Zhang et al. [33]. Elshehabey and Ahmed [34] analyzed effect of mixed convection in nanofluid flow with sinusoidal distribution of temperature on the both vertical walls using Buongiorno's nanofluid model.

The novelty of the present study is to account for the slip velocity, magnetic field, and mass transfer on squeezing unsteady nanofluid flow and heat transfer between two parallel plates. In this study, authors have applied Runge-KuttaFehlberg fourth-fifth-order method with shooting technique to find the solution of nonlinear differential equations. The effects of governing parameters such as slip, magnetic, and squeeze number, Schmidt number, and nanoparticle volume fraction on velocity, temperature, and concentration as well as on skin-friction coefficient, Nusselt, and Sherwood number are investigated. To the best of our best knowledge such investigation is not studied in the scientific literature. Some

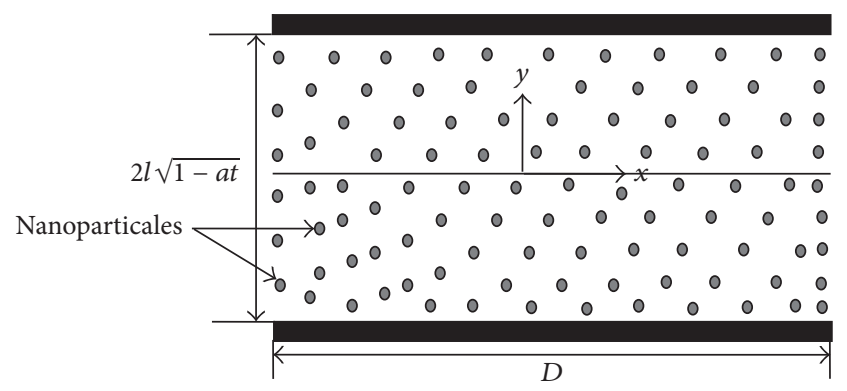

FIGURE 1: Flow configuration and coordinate system.

analytical methods for squeezing unsteady nanofluid flow can be found in $[4,6]$.

\section{Mathematical Formulation}

We consider an unsteady two-dimensional flow to observe heat and mass transfer of a squeezing nanofluid in the middle of two parallel plates extended infinitely and implanted in a system occupied with nanofluid (water as a base fluid) containing different types of nanoparticles, that is, copper $(\mathrm{Cu})$, silver $(\mathrm{Ag})$, alumina $\left(\mathrm{Al}_{2} \mathrm{O}_{3}\right)$, and titanium oxide $\left(\mathrm{TiO}_{2}\right)$ with slip velocity effect. The thermophysical properties of the nanofluids are given in Table 1. A transverse magnetic field of variable strength is imposed in direction perpendicular to both the plates. The distance between two plates is $z=$ $\pm l(1-\alpha t)^{1 / 2}= \pm h(t)$, where $l$ is the initial position (at time $t=0)$. Flow is incompressible with no chemical reaction in system. Further, viscous dissipation effects are retained. The graphical model support to the present study has been given in Figure 1. The governing equations representing flow are as follows:

$$
\begin{aligned}
& \frac{\partial u}{\partial x}+\frac{\partial v}{\partial y}=0 \\
& \rho_{\mathrm{nf}}\left(\frac{\partial u}{\partial t}+u \frac{\partial u}{\partial x}+v \frac{\partial u}{\partial y}\right) \\
& \quad=-\frac{\partial p}{\partial x}+\mu_{\mathrm{nf}}\left(\frac{\partial^{2} u}{\partial x^{2}}+\frac{\partial^{2} u}{\partial y^{2}}\right)-\sigma_{\mathrm{nf}} B_{0}^{2} u \\
& \rho_{\mathrm{nf}}\left(\frac{\partial v}{\partial t}+u \frac{\partial v}{\partial x}+v \frac{\partial v}{\partial y}\right)=-\frac{\partial p}{\partial y}+\mu_{\mathrm{nf}}\left(\frac{\partial^{2} v}{\partial x^{2}}+\frac{\partial^{2} v}{\partial y^{2}}\right)
\end{aligned}
$$




$$
\begin{aligned}
& \frac{\partial T}{\partial t}+ u \frac{\partial T}{\partial x}+v \frac{\partial T}{\partial y} \\
&= \frac{k_{\mathrm{nf}}}{\left(\rho C_{p}\right)_{\mathrm{nf}}}\left(\frac{\partial^{2} T}{\partial x^{2}}+\frac{\partial^{2} T}{\partial y^{2}}\right) \\
&+\frac{\mu_{\mathrm{nf}}}{\left(\rho C_{p}\right)_{\mathrm{nf}}}\left(4\left(\frac{\partial u}{\partial x}\right)^{2}+\left(\frac{\partial u}{\partial y}+\frac{\partial u}{\partial x}\right)^{2}\right), \\
& \frac{\partial C}{\partial t}+u \frac{\partial C}{\partial x}+v \frac{\partial C}{\partial y}=D\left(\frac{\partial^{2} C}{\partial x^{2}}+\frac{\partial^{2} C}{\partial y^{2}}\right) .
\end{aligned}
$$

The associated boundary conditions are given as

$$
\begin{aligned}
& u=-L \frac{\partial u}{\partial y}, \\
& v=v_{w}=\frac{d h}{d t}, \\
& T=T_{h}, \\
& C=C_{h} \text { at } y=h(t), \\
& v=\frac{\partial u}{\partial y}=\frac{\partial T}{\partial y}=0, \\
& C=C_{0} \quad \text { at } y=0,
\end{aligned}
$$

where

$$
\begin{aligned}
& \rho_{\mathrm{nf}}=(1-\varphi) \rho_{f}+\varphi \rho_{s}, \\
& \left(\rho C_{p}\right)_{\mathrm{nf}}=(1-\varphi)\left(\rho C_{p}\right)_{f}+\varphi\left(\rho C_{p}\right)_{s}, \\
& \mu_{\mathrm{nf}}=\frac{\mu_{f}}{(1-\varphi)^{2.5}}(\text { Brinkman), } \\
& \frac{k_{\mathrm{nf}}}{k_{f}}=\frac{k_{s}+2 k_{f}-2 \varphi\left(k_{f}-k_{s}\right)}{k_{s}+2 k_{f}+2 \varphi\left(k_{f}-k_{s}\right)} \text { (Maxwell-Garnetts), } \\
& \sigma_{\mathrm{nf}}=(1-\varphi) \sigma_{f}+\varphi \sigma_{s} .
\end{aligned}
$$

Equations (2)-(5) can be converted to a system of nonlinear ordinary differential equations via the following similarity variables:

$$
\begin{aligned}
& \eta=\frac{y}{l(1-\alpha t)^{1 / 2}}, \\
& u=\frac{\alpha x}{2(1-\alpha t)} f^{\prime}(\eta), \\
& v=-\frac{\alpha l}{2(1-\alpha t)^{1 / 2}} f(\eta), \\
& \theta=\frac{T-T_{0}}{T_{h}-T_{0}}, \\
& \phi=\frac{C-C_{0}}{C_{h}-C_{0}} .
\end{aligned}
$$

The transformed equations are

$$
\begin{aligned}
& f^{i v}-S A_{1}(1-\varphi)^{2.5}\left(\eta f^{\prime \prime \prime}+3 f^{\prime \prime}+f^{\prime} f^{\prime \prime}-f f^{\prime \prime \prime}\right) \\
& -M f^{\prime \prime}=0 \\
& \theta^{\prime \prime}+\operatorname{Pr} S\left(\frac{A_{2}}{A_{3}}\right)\left(\theta^{\prime} f-\eta \theta^{\prime}\right) \\
& \quad+\frac{\operatorname{PrEc}}{A_{3}(1-\varphi)^{2.5}}\left[f^{\prime \prime 2}+4 j^{2} f^{\prime 2}\right]=0, \\
& \phi^{\prime \prime}+\operatorname{ScS}\left(f \phi^{\prime}-\eta \phi^{\prime}\right)=0,
\end{aligned}
$$

where $A_{1}, A_{2}$, and $A_{3}$ are dimensionless constants defined as follows:

$$
\begin{aligned}
& A_{1}=(1-\varphi)+\varphi \frac{\rho_{s}}{\rho_{f}}, \\
& A_{2}=(1-\varphi)+\varphi \frac{\left(\rho C_{p}\right)_{s}}{\left(\rho C_{p}\right)_{f}}, \\
& A_{3}=\frac{k_{\mathrm{nf}}}{k_{f}} .
\end{aligned}
$$

The boundary conditions (6) in the terms of similarity variables (8) become

$$
\begin{aligned}
f(0) & =0, \\
f^{\prime \prime}(0) & =0, \\
\theta^{\prime}(0) & =0, \\
\phi(0) & =0, \\
f(1) & =1, \\
f^{\prime}(1) & =-\delta f^{\prime \prime}(1), \\
\theta(1) & =1, \\
\phi(1) & =1,
\end{aligned}
$$

where $S=\alpha l^{2} / 2 v_{f}$ is the squeeze number, $\operatorname{Pr}=\mu_{f}\left(\rho C_{p}\right)_{f} /$ $\rho_{f} k_{f}$ is the Prandtl number, Sc $=v_{f} / D_{\text {nf }}$ is Schmidt number, $M=2 \sigma_{\mathrm{nf}} B_{0}^{2}(h(t))^{2} / \rho_{f} \mu_{\mathrm{nf}}$ is the magnetic parameter, $j=l / x$ is the reference length and $\delta=L / l(1-\alpha t)^{1 / 2}$ is the velocity slip parameter, and Ec $=\left(\rho_{f} /\left(\rho C_{p}\right)_{f}\right)(\alpha x / 2(1-\alpha t))^{2}$ is the Eckert number.

The physical quantities of interest are the skin-friction coefficient $C_{f}$, the Nusselt number $\mathrm{Nu}_{x}$, and the Sherwood number $\mathrm{Sh}_{x}$ defined as

$$
\begin{aligned}
C_{f} & =\frac{\tau_{w}}{\rho_{\mathrm{nf}} v_{w}^{2}}, \\
\mathrm{Nu}_{x} & =\frac{l q_{w}}{k_{f}\left(T_{h}-T_{0}\right)}, \\
\mathrm{Sh}_{x} & =\frac{l m_{w}}{D_{\mathrm{nf}}\left(C_{h}-C_{0}\right)},
\end{aligned}
$$


where

$$
\begin{gathered}
\tau_{w}=\mu_{\mathrm{nf}}\left(\frac{\partial u}{\partial y}\right)_{y=h(t)}, \\
q_{w}=-k_{\mathrm{nf}}\left(\frac{\partial T}{\partial y}\right)_{y=h(t)}, \\
m_{w}=-D_{\mathrm{nf}}\left(\frac{\partial C}{\partial y}\right)_{y=h(t)} .
\end{gathered}
$$

Using (8) and (13) in (12), we get

$$
\begin{aligned}
C_{f}^{*} & =\frac{x^{2}(1-\alpha t) \operatorname{Re}_{x} C_{f}}{l^{2}}=\frac{f^{\prime \prime}(1)}{A_{1}(1-\phi)^{2.5}}, \\
\mathrm{Nu}_{x}^{*} & =\sqrt{1-\alpha t} \mathrm{Nu}_{x}=-A_{3} \theta^{\prime}(1), \\
\mathrm{Sh}_{x}{ }^{*} & =\sqrt{1-\alpha t} \mathrm{Sh}_{x}=-\phi^{\prime}(1),
\end{aligned}
$$

where $\operatorname{Re}_{x}=\alpha l^{5} / 2 x^{3}(1-\alpha t)^{1 / 2} v_{f}$ is the local Reynolds number.

\section{Method of Solution}

In this present paper, Runge-Kutta-Fehlberg fourth-fifthorder method (RKF45) has been employed to solve the system of nonlinear ordinary differential (9) with the boundary conditions given by (11) for different values of governing parameters. The RKF45 method has a procedure to determine if the suitable step size $h$ is being used. The formula of fifth-order Runge-Kutta-Fehlberg method can be defined as follows:

$$
\begin{aligned}
& z_{n+1}=z_{n} \\
& +\left(\frac{16}{135} k_{0}+\frac{6656}{12825} k_{2}+\frac{28561}{56430} k_{3}-\frac{9}{50} k_{4}+\frac{2}{55} k_{5}\right) \\
& . h,
\end{aligned}
$$

where the coefficients $k_{0}$ to $k_{5}$ are defined as follows:

$$
\begin{aligned}
k_{0} & =f\left(x_{n}, y_{n}\right), \\
k_{1} & =f\left(x_{n}+\frac{1}{4} h, y_{n}+\frac{1}{4} h k_{0}\right), \\
k_{2} & =f\left(x_{n}+\frac{3}{8} h, y_{n}+\left(\frac{3}{32} k_{0}+\frac{9}{32} k_{1}\right) h\right), \\
k_{3} & =f\left(x_{n}+\frac{12}{13} h, y_{n}\right. \\
& \left.+\left(\frac{1932}{2197} k_{0}-\frac{7200}{2197} k_{1}+\frac{7296}{2197} k_{2}\right) h\right),
\end{aligned}
$$

$$
\begin{aligned}
k_{4} & =f\left(x_{n}+h, y_{n}\right. \\
& \left.+\left(\frac{439}{216} k_{0}-8 k_{1}+\frac{3680}{513} k_{2}-\frac{845}{4104} k_{3}\right) h\right), \\
k_{5} & =f\left(x_{n}+\frac{1}{2} h, y_{n}\right. \\
& \left.+\left(-\frac{8}{27} k_{0}+2 k_{1}-\frac{3544}{2565} k_{2}+\frac{1859}{4104} k_{3}-\frac{11}{40} k_{4}\right) h\right) .
\end{aligned}
$$

The computation of the error can be achieved by subtracting the fifth-order from the fourth-order method,

$$
y_{n+1}=y_{n}+\left(\frac{25}{216} k_{0}+\frac{1408}{2565} k_{2}+\frac{2197}{4104} k_{3}-\frac{1}{5} k_{4}\right) h .
$$

If the error goes beyond a specified antechamber, the results can be recalculated using a smaller step size. The approach to computing the new step size is shown as follows:

$$
h_{\text {new }}=h_{\text {old }}\left(\frac{\varepsilon h_{\text {old }}}{2\left|z_{n+1}-y_{n+1}\right|}\right)^{1 / 4} \text {. }
$$

The variation of the dimensionless velocity, temperature, and concentration is ensured to be less than $10^{-6}$ between any two consecutive iterations for the convergence criterion.

To solve the nonlinear differential equations (9) subject to the boundary conditions (11), first boundary conditions for $\eta=1$ are replaced by $f(1)=1, f^{\prime}(1)=-\delta f^{\prime \prime}(1), \theta(1)=1$, and $\phi(1)=1$. We consider that $f=f_{1}, f^{\prime}=f_{2}, f^{\prime \prime}=$ $f_{3}, f^{\prime \prime \prime}=f_{4}, \theta=f_{5}, \theta^{\prime}=f_{6}, \phi=f_{7}$, and $\phi^{\prime}=f_{8}$. The nonlinear equations (9) are first converted into first-order ordinary linear differential equations as follows:

$$
\begin{aligned}
f^{\prime}= & f_{2}, \\
f_{2}^{\prime}= & f_{3}, \\
f_{3}^{\prime}= & f_{4}, \\
f_{4}^{\prime}= & S A_{1}(1-\varphi)^{2.5}\left(\eta f_{4}+3 f_{3}+f_{2} f_{3}-f_{1} f_{4}\right)+M f_{3}, \\
f_{5}^{\prime}= & f_{6}, \\
f_{6}^{\prime}= & -\operatorname{Pr} S\left(\frac{A_{2}}{A_{3}}\right)\left(f_{6} f_{1}-\eta f_{6}\right) \\
& -\frac{\operatorname{PrEc}}{A_{3}(1-\varphi)^{2.5}}\left[f_{3}^{2}+4 j^{2} f_{2}^{2}\right], \\
f_{7}^{\prime}= & f_{8}, \\
f_{8}^{\prime}= & -S c S\left(f_{1} f_{8}-\eta f_{8}\right),
\end{aligned}
$$


TABLE 2: Comparison of $f(\eta)$ and $\theta(\eta)$ for Cu-water nanofluid when $S=1, \operatorname{Pr}=6.2$, Ec $=j=0.01, \varphi=0.02$, and $M=\mathrm{Sc}=\delta=0$.

\begin{tabular}{|c|c|c|c|c|}
\hline \multirow{2}{*}{$\eta$} & \multicolumn{2}{|c|}{ Gupta and Ray [4] } & \multicolumn{2}{|c|}{ Present result } \\
\hline & $f(\eta)$ & $\theta(\eta)$ & $f(\eta)$ & $\theta(\eta)$ \\
\hline 0 & 0 & 1.03206637 & 0 & 1.02996637 \\
\hline 0.1 & 0.14135866 & 1.03206407 & 0.14135879 & 1.02996428 \\
\hline 0.2 & 0.28066605 & 1.03203202 & 0.28066639 & 1.02993531 \\
\hline 0.3 & 0.41578075 & 1.03189263 & 0.41578137 & 1.02980932 \\
\hline 0.4 & 0.54437882 & 1.03150811 & 0.54437979 & 1.02946175 \\
\hline 0.5 & 0.66385692 & 1.03066423 & 0.66385837 & 1.02869896 \\
\hline 0.6 & 0.77122923 & 1.02903983 & 0.77123132 & 1.02723066 \\
\hline 0.7 & 0.86301562 & 1.02615205 & 0.86301853 & 1.02462037 \\
\hline 0.8 & 0.93511971 & 1.02125916 & 0.93512364 & 1.02019765 \\
\hline 0.9 & 0.98269524 & 1.01318608 & 0.98270044 & 1.01290031 \\
\hline 1.0 & 1.00000000 & 1.00000000 & 1.00000000 & 1.00000000 \\
\hline
\end{tabular}

subject to the following initial conditions:

$$
\begin{aligned}
& f_{1}(0)=0, \\
& f_{2}(0)=\alpha_{1}, \\
& f_{3}(0)=0, \\
& f_{4}(0)=\alpha_{2}, \\
& f_{5}(0)=\alpha_{3}, \\
& f_{6}(0)=0, \\
& f_{7}(0)=0, \\
& f_{8}(0)=\alpha_{4} .
\end{aligned}
$$

We ran the computer code written in the MATLAB for different values of step length $\nabla \eta$. We found that there is no or only a negligible change in the physical quantities of interest like the skin-friction coefficient, the couple stress coefficient, Nusselt number, and Sherwood number for various values of $\nabla \eta>0.01$. Therefore, in the present paper we have set step size $\nabla \eta=0.01$. There are four initial conditions at $\eta=0$ and four conditions on boundary $\eta=1$. To get the solution of the problem, four more initial conditions at $\eta=0$, that is, values of $\alpha_{1}, \alpha_{2}, \alpha_{3}$, and $\alpha_{4}$, are required, which have been obtained by shooting technique. Finally the transformed initial value problem is solved by employing the Runge-Kutta-Fehlberg fourth-fifth-order method along with calculated boundary conditions.

\section{Results and Discussion}

In order to validate the numerical results obtained, we compare our results with those reported by Gupta and Ray [4] as shown in Table 2, and they are found to be in a good agreement. The effects of the volume fraction of solid nanoparticles, magnetic parameter, velocity slip parameter, squeeze number, and Schmidt number are inspected for different kinds of nanoparticles when the base fluid is water, $\mathrm{Ec}=0.01, \operatorname{Pr}=6.2$, and $j=0.01$.

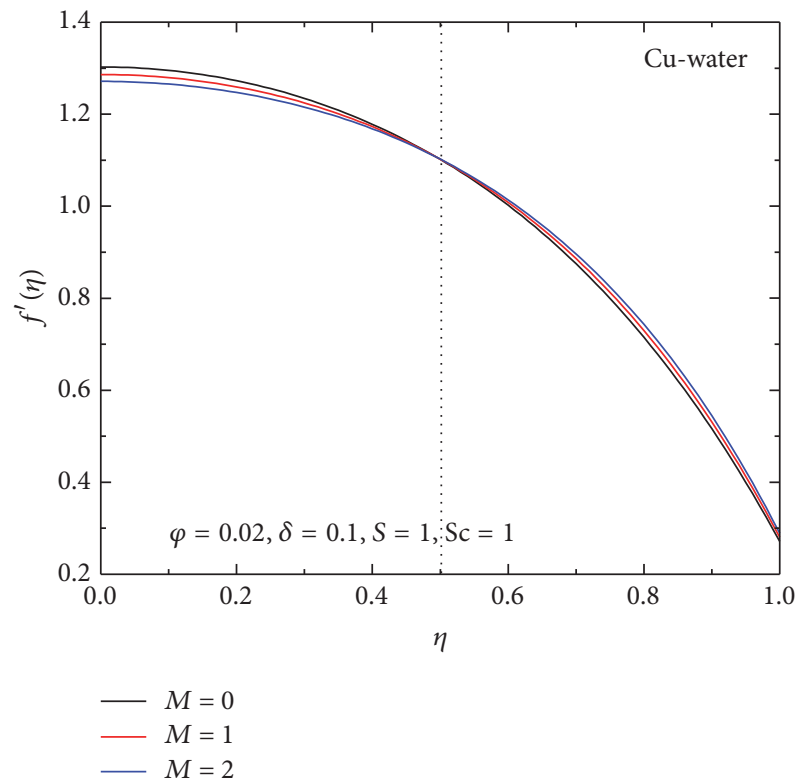

Figure 2: Effects of $M$ on velocity $f^{\prime}(\eta)$.

Figures 2 and 3 illustrate the behavior of the velocity $f^{\prime}(\eta)$ and temperature $\theta(\eta)$ of the $\mathrm{Cu}$-water nanofluid for different values of magnetic parameter $M$. Figure 2 shows that, with an increase in the values of $M$, the velocity decreases near the lower plate surface, but after a certain distance it increases. It is noticed from Figure 3 that the temperature decreases monotonically with increasing values of $M$.

The variations of the velocity, temperature, and concentration for different values of slip parameter $\delta$ are shown in Figures $4-6$. It is noted from Figure 4 that near the wall the value of velocity $f^{\prime}(\eta)$ increases with rising values of $\delta$ when $0 \leq \eta \leq 0.6$ and for $\eta \geq 0.6$ the velocity decreases as $\delta$ increases. Figure 5 depicts that a rise in the values of slip parameter $\delta$ increases temperature monotonically. It is evident from Figure 6 that the change in the concentration $\phi(\eta)$ with rising values of slip parameter $\delta$ is negligible. 


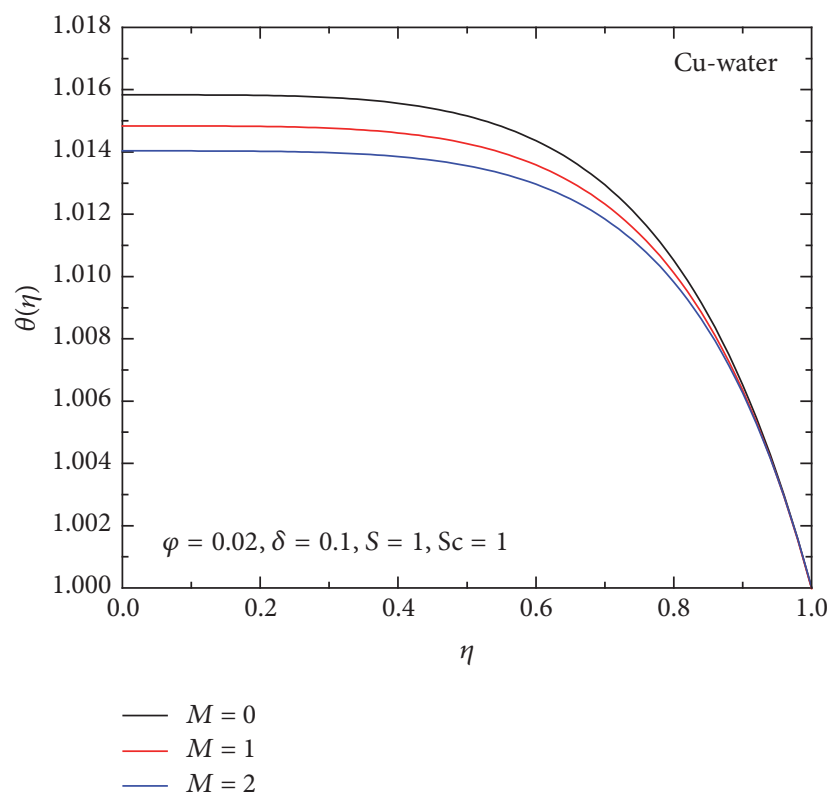

Figure 3: Effects of $M$ on temperature $\theta(\eta)$.

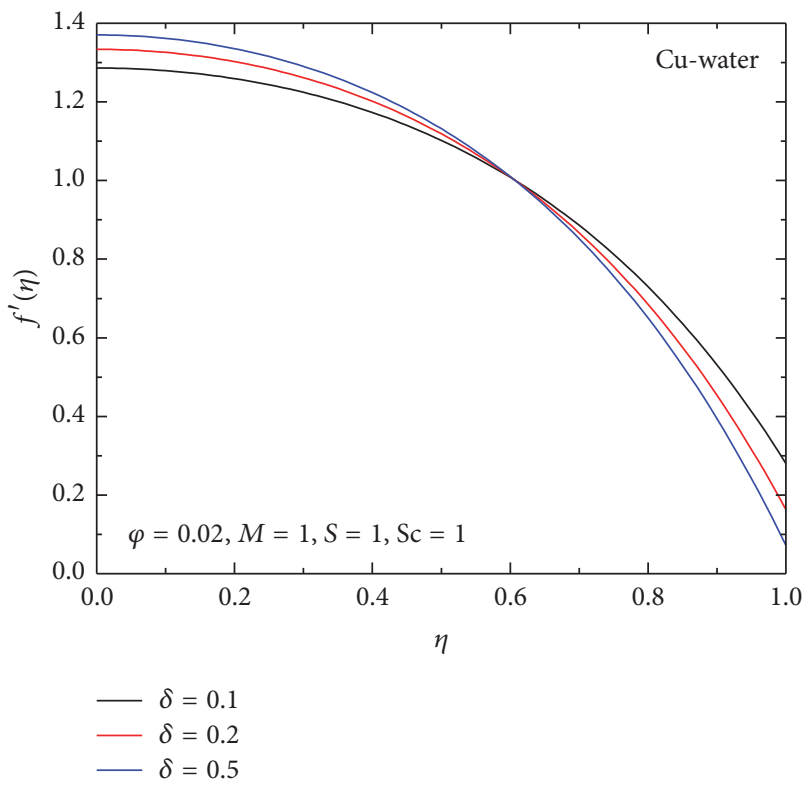

FIgURE 4: Effects of $\delta$ on velocity $f^{\prime}(\eta)$.

The effects of the squeeze number $S$ on velocity, temperature, and concentration profiles are depicted in Figures 7-9. Physically the squeeze number $(S)$ describes the movement of the plates $(S>0$ corresponds to the plates moving apart, while $S<0$ corresponds to the plates moving together). It can be easily seen from Figure 7 that the value of velocity $f^{\prime}(\eta)$ near the lower plate surface decreases regularly with the increase in the value of $S$, and as we move away from lower plate surface this value increases. Figure 8 shows that increasing value of squeeze number $S$ decreases the temperature $\theta(\eta)$. Figure 9 depicts that the value of $\phi(\eta)$ goes

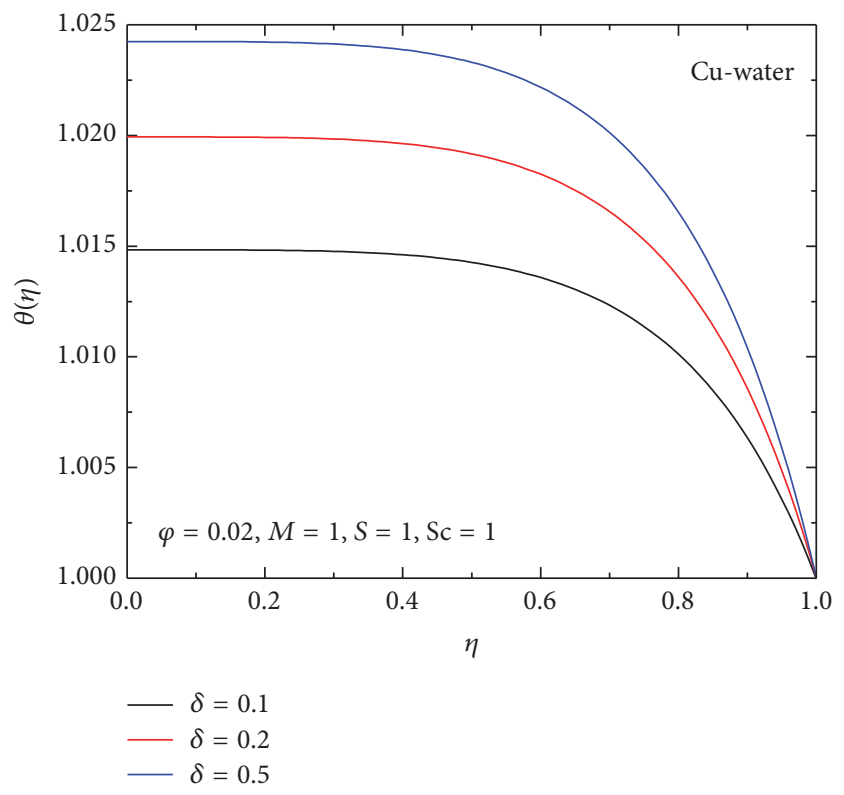

Figure 5: Effects of $\delta$ on temperature $\theta(\eta)$.

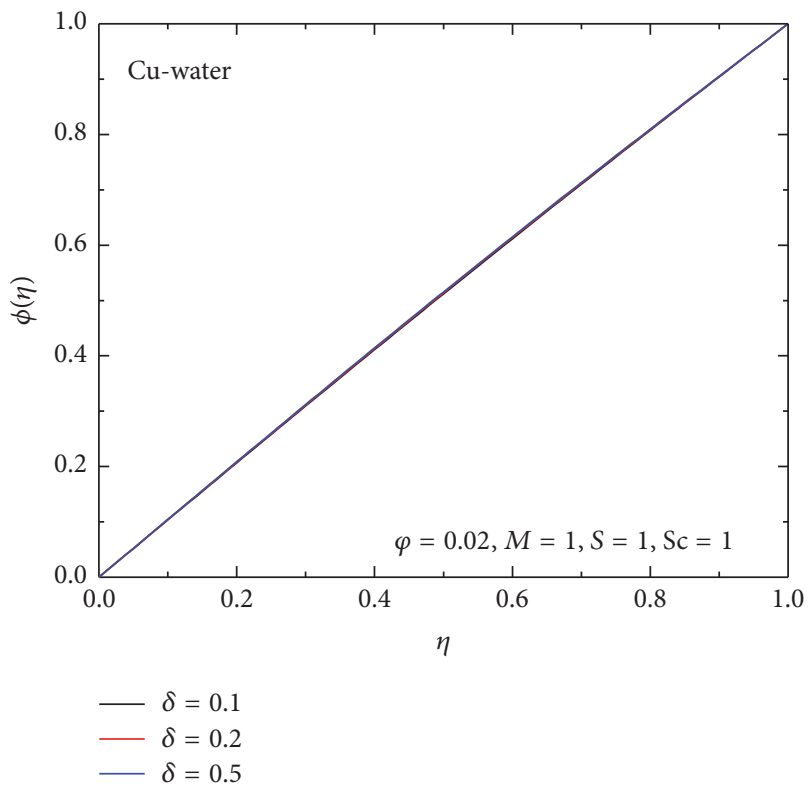

FiguRE 6: Effects of $\delta$ on concentration $\phi(\eta)$.

up with increasing $\eta$ and high squeeze number implies slight drop in concentration $\phi(\eta)$.

Figures 10 and 11 show the effects of the volume fraction $\varphi$ on the velocity and temperature profiles. Figure 10 exhibits initially an increase in the values of nanoparticles volume fraction $\varphi$, the velocity $f^{\prime}(\eta)$ decreases, and after a fixed distance from lower plate surface it increases slightly. From Figure 11, it is observed that the temperature decreases with increasing values of $\varphi$.

Figures 12 and 13 illiterate the effect of the different nanoparticles on velocity and temperature profile when the base fluid is water. It is observed from Figure 12 that 


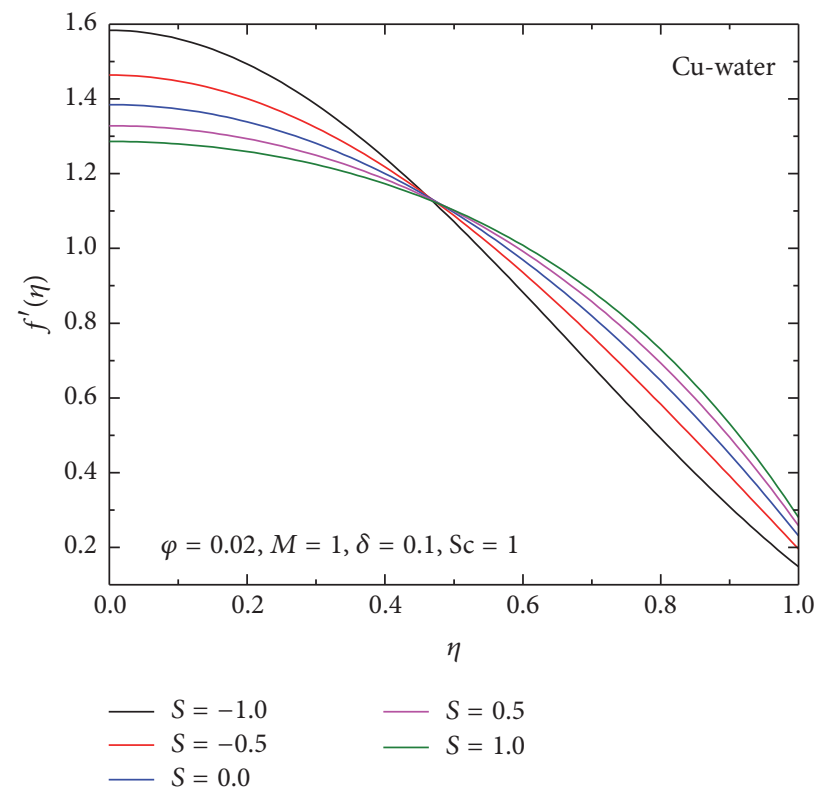

Figure 7: Effects of $S$ on velocity $f^{\prime}(\eta)$.

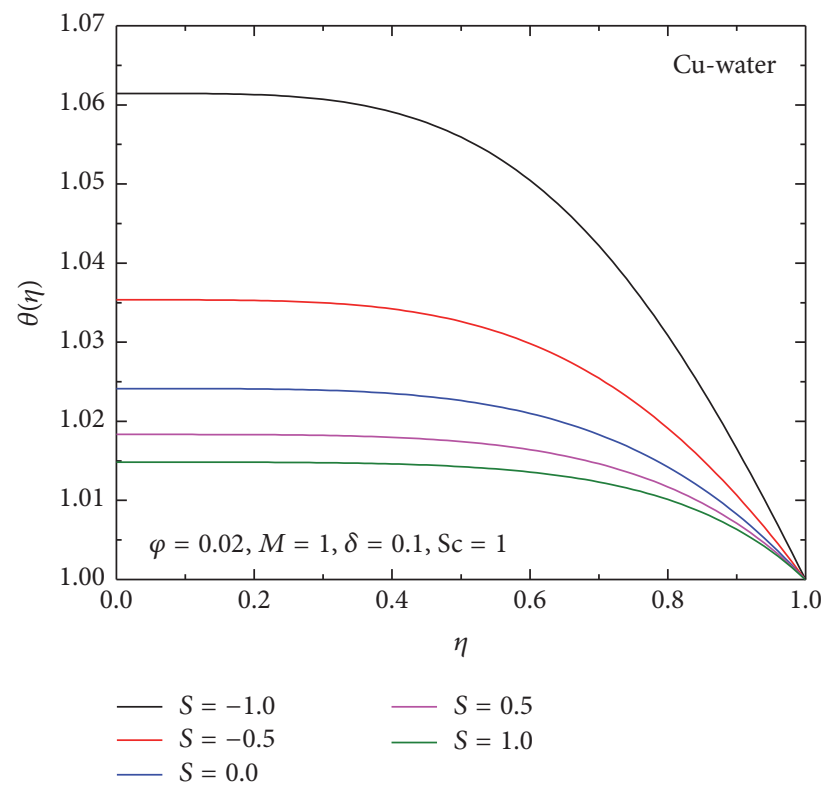

Figure 8: Effects of $S$ on temperature $\theta(\eta)$.

the different nanofluids have different velocities and also noted that titanium oxide $\left(\mathrm{TiO}_{2}\right)$ has higher velocity when compared to other nanoparticles such as $\mathrm{Al}_{2} \mathrm{O}_{3}, \mathrm{Cu}$, and Ag for $0 \leq \eta \leq 0.5$, while for $\eta>0.5$ the results get reversed. It is noted from Figure 13 that the order of nanofluid for decreasing value of $\theta(\eta)$ when $\eta$ change from 0 to 1 is $\mathrm{TiO}_{2}$-water, $\mathrm{Al}_{2} \mathrm{O}_{3}$-water, $\mathrm{Cu}$-water, and $\mathrm{Ag}$-water nanofluid. Figure 14 depicts the effect of the Schmidt number Sc on concentration profile. The values of the temperature increase with the increase in the value of Sc.

The effects of the squeeze number $S$ on the skin-friction coefficient $C_{f}{ }^{*}$, the Nusselt number $\mathrm{Nu}_{x}{ }^{*}$, and the Sherwood

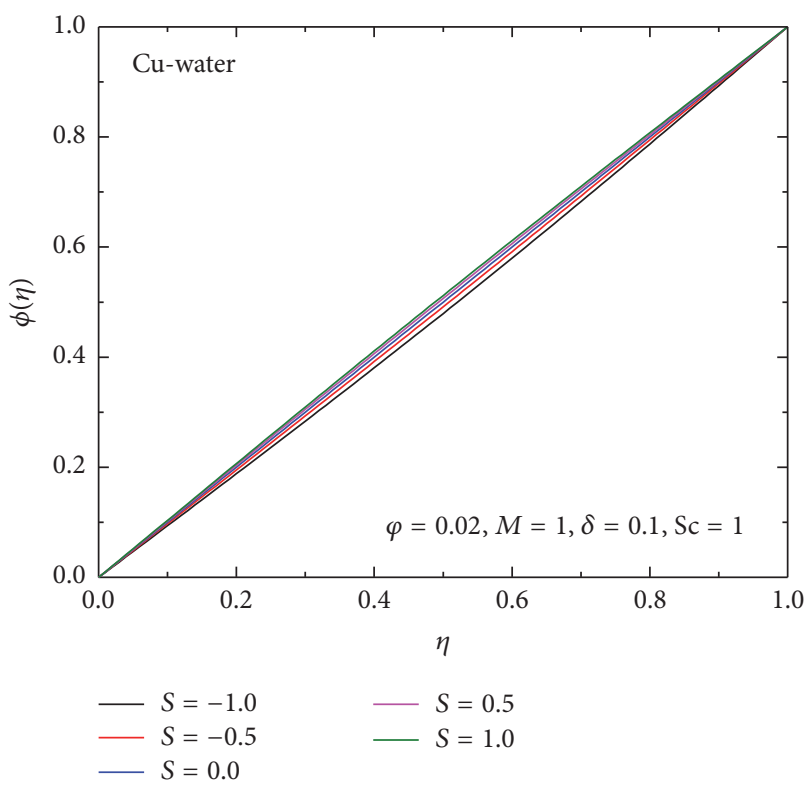

Figure 9: Effects of $S$ on concentration $\phi(\eta)$.

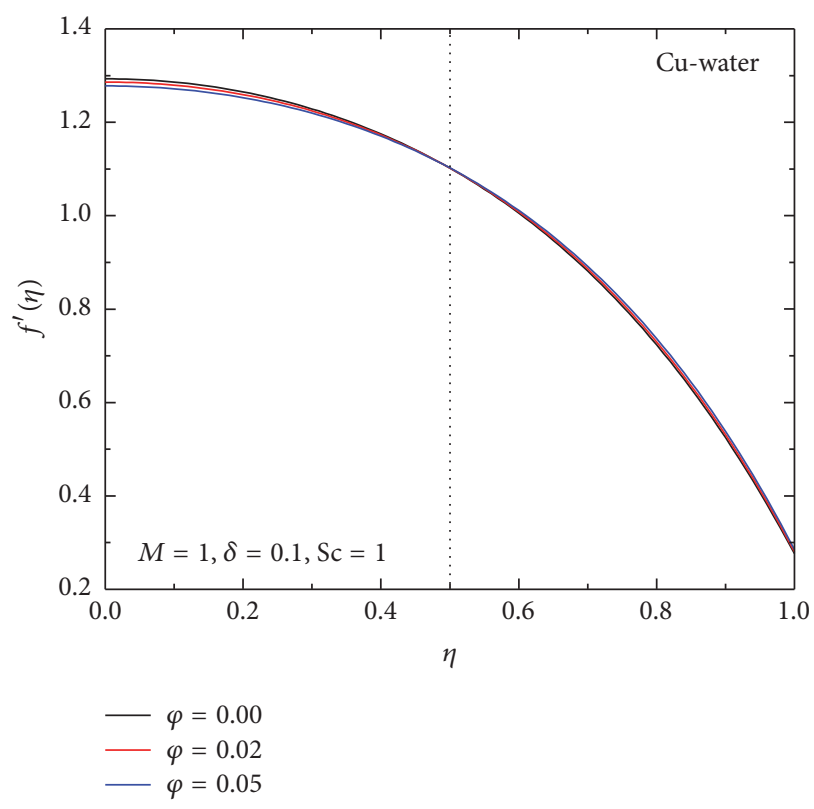

FIGURE 10: Effects of $\varphi$ on velocity $f^{\prime}(\eta)$.

number $\mathrm{Sh}_{x}{ }^{*}$ are given in Table 3. From Table 3, it is obvious that the skin-friction coefficient and the Nusselt number are inversely proportional to $S$, whereas the Sherwood number is directly proportional to $S$.

Table 4 displays the effects of the skin-friction coefficient, the Nusselt number, and Sherwood number for different values of the magnetic parameter $M$ and slip parameter $\delta$. It is noticed from the table that the effect of increasing values of $M$ is to increase the skin-friction coefficient $C_{f}{ }^{*}$, the heat transfer rate $\mathrm{Nu}_{x}{ }^{*}$, and mass transfer rate $\mathrm{Sh}_{x}{ }^{*}$. Further, from Table 4 it is concluded that the increasing value of $\delta$ decreases 


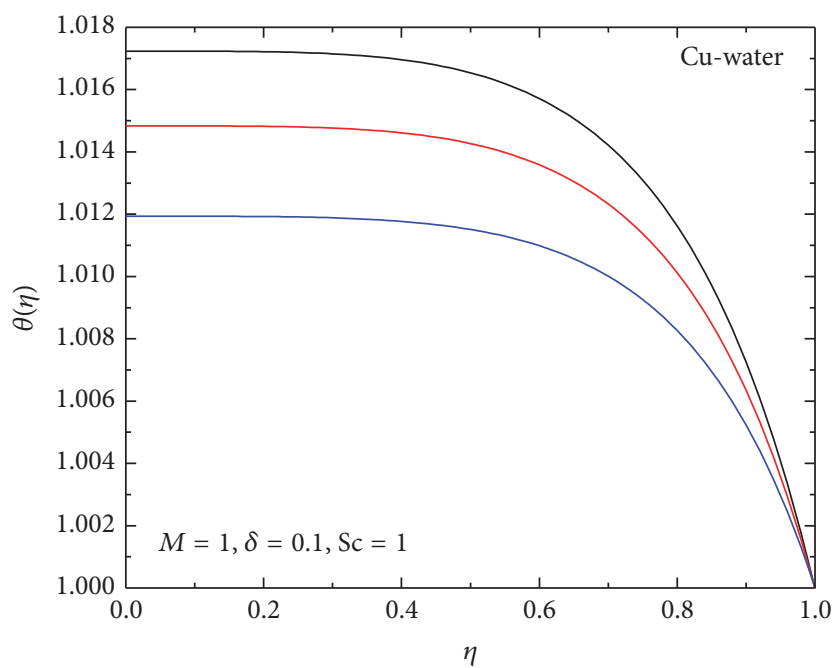

$\begin{aligned}-\varphi & =0.00 \\ \varphi & =0.02 \\ \varphi & =0.05\end{aligned}$

Figure 11: Effects of $\varphi$ on temperature $\theta(\eta)$.

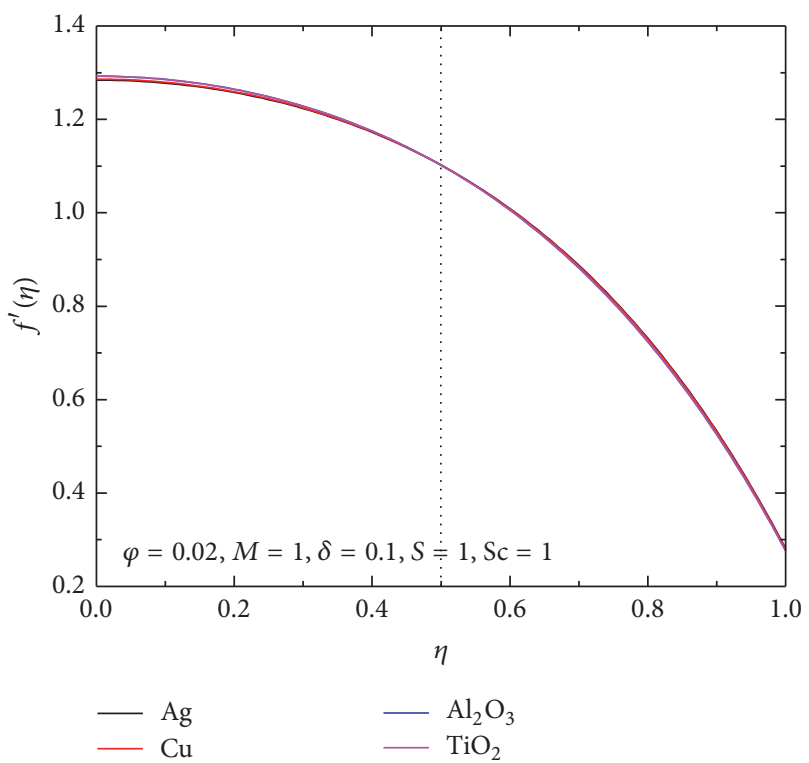

FIGURE 12: Velocity of different nanofluids.

the skin-friction coefficient and increases the heat and mass transfer rate.

The effects of the nanoparticle volume fraction $\varphi$ and Schmidt number Sc on the skin-friction coefficient $C_{f}{ }^{*}$, Nusselt number (the heat transfer rate) $\mathrm{Nu}_{x}{ }^{*}$, and Sherwood number (the mass transfer rate) $\mathrm{Sh}_{x}{ }^{*}$ are given in Table 5. From this table it is concluded that the increasing value of $\varphi$ increases the skin-friction coefficient and decreases the heat and mass transfer rate. In addition, the rate of mass transfer increases with the increase in Sc, but there is no effect of Sc on the skin-friction coefficient and the heat transfer rate.

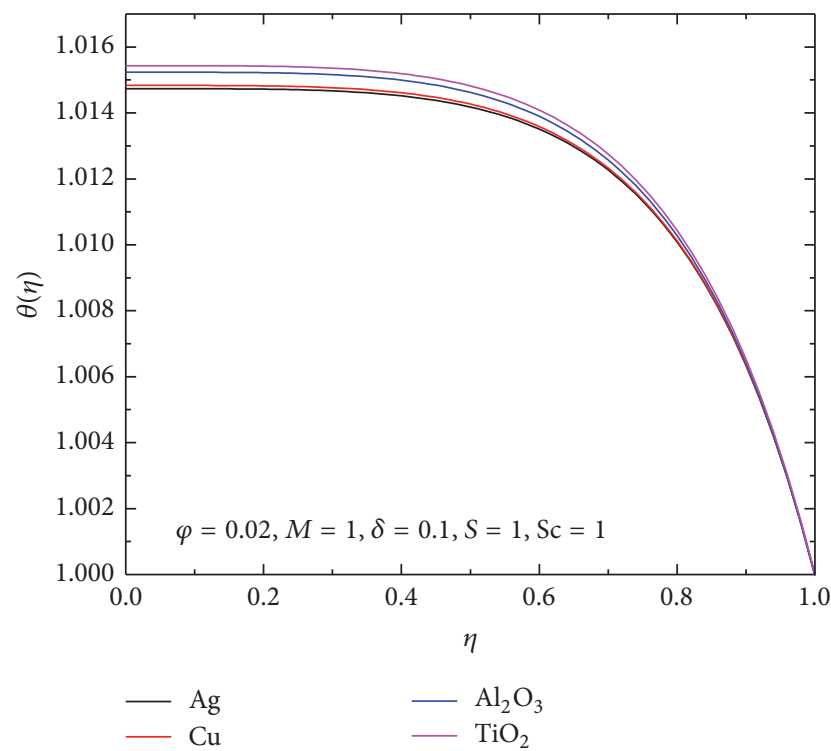

FIGURE 13: Temperature of different nanofluids.

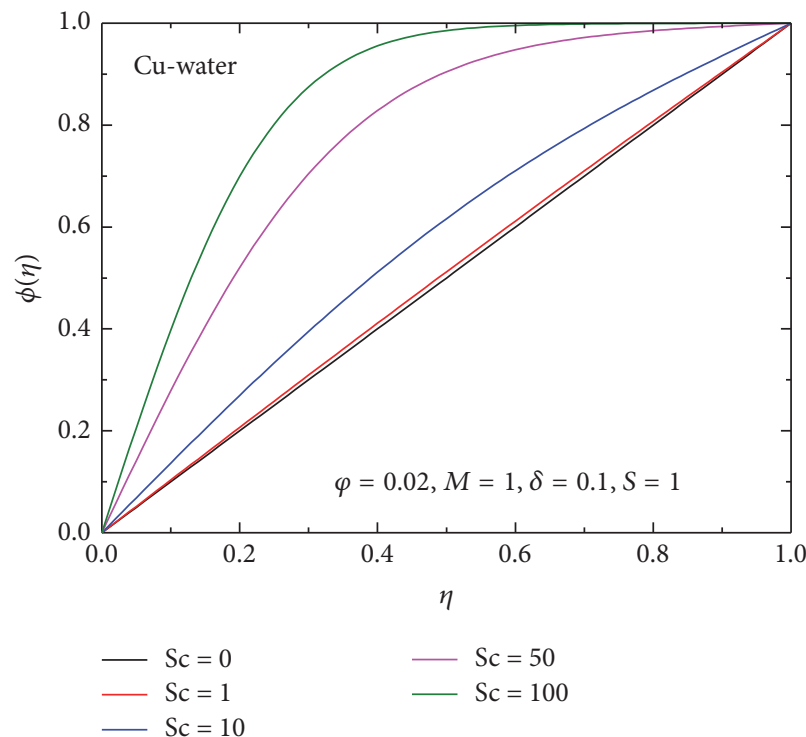

FIGURE 14: Effects of Sc on concentration $\phi(\eta)$

TABLE 3: Variation of $C_{f}{ }^{*}, \mathrm{Nu}_{x}{ }^{*}$, and $\mathrm{Sh}_{x}{ }^{*}$ with different values of Squeeze number $S$ for $\mathrm{Cu}$-water nanofluid when $M=1, \mathrm{Sc}=$ $1, \delta=0.1$, and $\varphi=0.02$.

\begin{tabular}{lccc}
\hline$S$ & $C_{f}{ }^{*}$ & $\mathrm{Nu}_{x}{ }^{*}$ & $\mathrm{Sh}_{x}{ }^{*}$ \\
\hline-1.0 & -1.34295 & 0.187977 & -1.0694254 \\
-0.5 & -1.77787 & 0.127657 & -1.0293233 \\
0.0 & -2.09419 & 0.104693 & -1.0000000 \\
0.5 & -2.34148 & 0.094096 & -0.9770841 \\
1.0 & -2.54426 & 0.088577 & -0.9583107 \\
\hline
\end{tabular}

The comparison for metallic nanoparticles ( $\mathrm{Ag}, \mathrm{Cu})$ and nonmetallic nanoparticles $\left(\mathrm{Al}_{2} \mathrm{O}_{3}, \mathrm{TiO}_{2}\right)$ is done in 
TABLE 4: Variation of $C_{f}{ }^{*}, \mathrm{Nu}_{x}{ }^{*}$, and $\mathrm{Sh}_{x}{ }^{*}$ with different values of magnetic parameter $M$ and slip parameter $\delta$ for $\mathrm{Cu}$-water nanofluid when $S=1, \mathrm{Sc}=1$, and $\varphi=0.02$.

\begin{tabular}{lcccc}
\hline$M$ & $\delta$ & $C_{f}{ }^{*}$ & $\mathrm{Nu}_{x}{ }^{*}$ & $\mathrm{Sh}_{x}{ }^{*}$ \\
\hline 0 & 0.1 & -2.46084 & 0.089198 & -0.95676 \\
1 & 0.1 & -2.54426 & 0.088577 & -0.95831 \\
2 & 0.1 & -2.6223 & 0.088075 & -0.95974 \\
1 & 0.2 & -2.95257 & 0.118598 & -0.95158 \\
1 & 0.5 & -3.26534 & 0.144432 & -0.94633 \\
\hline
\end{tabular}

TABLE 5: Variation of $C_{f}{ }^{*}, \mathrm{Nu}_{x}{ }^{*}$, and $\mathrm{Sh}_{x}{ }^{*}$ with different values of volume fraction $\varphi$ and Schmidt number Sc for Cu-water nanofluid when $M=1, S=1$, and $\delta=0.1$.

\begin{tabular}{lcccr}
\hline$\varphi$ & $\mathrm{Sc}$ & $C_{f}{ }^{*}$ & $\mathrm{Nu}_{x}{ }^{*}$ & $\mathrm{Sh}_{x}{ }^{*}$ \\
\hline 0.00 & 1 & -2.76550 & 0.092885 & -0.95773 \\
0.02 & 1 & -2.54426 & 0.088577 & -0.95831 \\
0.05 & 1 & -2.31844 & 0.082295 & -0.95911 \\
0.02 & 0 & -2.54426 & 0.088577 & -1.00000 \\
0.02 & 10 & -2.54426 & 0.088577 & -0.63318 \\
0.02 & 50 & -2.54426 & 0.088577 & -0.05952 \\
0.02 & 100 & -2.54426 & 0.088577 & -0.00182 \\
\hline
\end{tabular}

TABLE 6: Variation of $C_{f}{ }^{*}, \mathrm{Nu}_{x}{ }^{*}$, and $\mathrm{Sh}_{x}{ }^{*}$ for different nanoparticles when $M=1, S=1, \mathrm{Sc}=1, \delta=0.1$, and $\varphi=0.02$.

\begin{tabular}{lccc}
\hline Nanoparticle & $C_{f}{ }^{*}$ & $\mathrm{Nu}_{x}{ }^{*}$ & $\mathrm{Sh}_{x}{ }^{*}$ \\
\hline $\mathrm{Ag}$ & -2.48678 & 0.088535 & -0.95851 \\
$\mathrm{Cu}$ & -2.54426 & 0.088577 & -0.95831 \\
$\mathrm{TiO}_{2}$ & -2.73607 & 0.088742 & -0.95777 \\
$\mathrm{Al}_{2} \mathrm{O}_{3}$ & -2.74807 & 0.088794 & -0.95772 \\
\hline
\end{tabular}

Table 6. It is observed form Table 6 that value of the skinfriction coefficient is more for the metallic nanoparticles than nonmetallic nanoparticles, whereas the nonmetallic nanoparticles have higher heat and mass transfer rate in comparison with metallic nanoparticles.

\section{Conclusions}

The present paper deals with the numerical solution of combined heat and mass transfer effects of an unsteady MHD laminar two-dimensional flow of incompressible viscous nanofluids in middle of two parallel plates extended infinitely with slip velocity effect. The relevant nonlinear partial differential equations were transformed to a set of ordinary differential equations and then are solved numerically using the Runge-Kutta-Fehlberg fourth-fifth-order method along with shooting technique. From the above discussion the eyecatching results are as follows:

(i) Temperature drops in $\mathrm{Cu}$-water nanofluid with increase in the magnetic field strength.

(ii) The velocity of the nanofluid with nonmetallic nanoparticles $\mathrm{Al}_{2} \mathrm{O}_{3}$ and $\mathrm{TiO}_{2}$ is greater than metallic nanoparticles $\mathrm{Cu}$ and $\mathrm{Ag}$ initially when water is the base fluid, but nature gets reversed after $\eta=0.5$. The temperature of the metallic nanoparticles is lower than the nonmetallic nanoparticles.

(iii) When plates start moving apart, then temperature starts decreasing.

(iv) Initially, with the increasing values of magnetic field strength and squeeze number, the velocity of $\mathrm{Cu}$ water slows down but the nature in both the case gets reversed after $\eta=0.5$.

(v) The Nusselt number and skin-friction coefficient decrease as plates move apart for $\mathrm{Cu}$-water nanofluid.

(vi) Nusselt number and Sherwood number for nonmetallic nanoparticles are higher than the metallic nanoparticles.

(vii) Varying value of slip parameter has negligible effect on concentration and as values of squeeze number increase, the value of concentration profile also gets slightly higher.

(viii) Considerable amount of enhancement in concentration can be seen as Schmidt number rises.

\section{Nomenclature}

$A_{1}, A_{2}, A_{3}$ : Dimensionless constant

$B_{0}: \quad$ Strength of magnetic field

C: $\quad$ Fluid concentration

$C_{f}: \quad$ Skin-friction coefficient

$C_{p}$ : $\quad$ Specific heat at constant pressure $p\left[\mathrm{Jkg}^{-1} \mathrm{~K}^{-1}\right]$

D: $\quad$ Mass diffusivity 
Ec: Eckert number

$f$ : Dimensionless velocity of the fluid

$j$ : $\quad$ Reference length

$k_{\text {nf }}$ : Nanofluid effective thermal conductivity

l: $\quad$ Distance of plate $[\mathrm{m}]$

$L: \quad$ Slip length

M: Magnetic parameter

$m_{w}$ : Surface mass flux

$\mathrm{Nu}_{x}$ : Nusselt number

$p: \quad$ Pressure $[\mathrm{Pa}]$

Pr: Prandtl number

$q_{w}:$ Surface heat flux

$\mathrm{Re}_{x}$ : Local Reynolds number

$S$ : $\quad$ Squeeze number

Sc: Schmidt number

$\mathrm{Sh}_{x}$ : Sherwood number

$t$ : Time [s]

$T: \quad$ Temperature $[\mathrm{K}]$

$u, v$ : Velocities in $x y$-directions $\left[\mathrm{m} \mathrm{s}^{-1}\right]$

$x, y$ : Axial and perpendicular coordinates [m].

\section{Greek Symbols}

$\mu_{f}:$ Dynamic viscosity of the fluid

$\varphi$ : Nanoparticle volume fraction

$\delta$ : Velocity slip parameter

$v_{f}$ : Kinematic viscosity of the fluid $\left[\mathrm{m}^{2} \mathrm{~s}^{-1}\right]$

$\eta$ : Similarity variable

$\rho_{f}$ : Density of base fluid $\left[\mathrm{kg} \mathrm{m}^{-3}\right]$

$k_{s}$ : Thermal conductivity of the solid nanoparticle $\left[\mathrm{m}^{-1} \mathrm{~K}^{-1}\right]$

$k_{f}$ : Thermal conductivity of the base fluid $\left[\mathrm{Wm}^{-1} \mathrm{~K}^{-1}\right.$ ]

$\theta$ : Nondimensional temperature

$\phi$ : Nondimensional concentration

$\rho_{\mathrm{nf}}:$ Nanofluid density $\left[\mathrm{kg} \mathrm{m}^{-3}\right]$

$\mu_{\mathrm{nf}}$ : Effective dynamic viscosity of nanofluid [Pa s]

$\alpha$ : Squeeze parameter

$\sigma_{\mathrm{nf}}$ : Electrical conductivity of nanofluid

$\rho_{s}$ : Density of solid particle $\left[\mathrm{kg} \mathrm{m}^{-3}\right]$

$\tau_{w}$ : Surface shear stress.

\section{Subscripts}
$f$ : Base fluid
$h$ : Condition at the wall
nf: Nanofluid
$s$ : Solid.

\section{Competing Interests}

The authors declare that there is no conflict of interests regarding the publication of this paper.

\section{References}

[1] M. J. Stefan, "Versuchüber die scheinbare adhesion sitzungsbersächs," Akademie der Wissenschaften in Wien. Mathematisch-Naturwissenschaftliche Klasse, vol. 69, pp. 713-721, 1874.

[2] G. Domairry and M. Hatami, "Squeezing Cu-water nanofluid flow analysis between parallel plates by DTM-Padé Method," Journal of Molecular Liquids, vol. 193, pp. 37-44, 2014.

[3] O. Pourmehran, M. Rahimi-Gorji, M. Gorji-Bandpy, and D. D. Ganji, "Analytical investigation of squeezing unsteady nanofluid flow between parallel plates by LSM and CM," Alexandria Engineering Journal, vol. 54, no. 1, pp. 17-26, 2015.

[4] A. K. Gupta and S. S. Ray, "Numerical treatment for investigation of squeezing unsteady nanofluid flow between two parallel plates," Powder Technology, vol. 279, pp. 282-289, 2015.

[5] U. Khan, N. Ahmed, M. Asadullah, and S. T. Mohyud-din, "Effects of viscous dissipation and slip velocity on two-dimensional and axisymmetric squeezing flow of $\mathrm{Cu}$-water and $\mathrm{Cu}$ kerosene nanofluids," Propulsion and Power Research, vol. 4, no. 1, pp. 40-49, 2015.

[6] A. Dib, A. Haiahem, and B. Bou-said, "Approximate analytical solution of squeezing unsteady nanofluid flow," Powder Technology, vol. 269, pp. 193-199, 2015.

[7] S. U. S. Choi and J. A. Eastman, "Enhancing thermal conductivity of fluids with nanoparticle," in Proceedings of the International Mechanical Engineering Congress and Exhibition, vol. 231, pp. 99-105, San Francisco, Calif, USA, November 1995.

[8] M. Azimi and R. Riazi, "Heat transfer analysis of GO-water nanofluid flow between two parallel disks," Propulsion and Power Research, vol. 4, no. 1, pp. 23-30, 2015.

[9] A. Aziz, W. A. Khan, and I. Pop, "Free convection boundary layer flow past a horizontal flat plate embedded in porous medium filled by nanofluid containing gyrotactic microorganisms," International Journal of Thermal Sciences, vol. 56, pp. 4857, 2012.

[10] M. Fakour, D. D. Ganji, and M. Abbasi, "Scrutiny of underdeveloped nanofluid MHD flow and heat conduction in a channel with porous walls," Case Studies in Thermal Engineering, vol. 4, pp. 202-214, 2014.

[11] T. Groşan, C. Revnic, I. Pop, and D. B. Ingham, "Free convection heat transfer in a square cavity filled with a porous medium saturated by a nano-fluid," International Journal of Heat and Mass Transfer, vol. 87, pp. 36-41, 2015.

[12] A. V. Kuznetsov and D. A. Nield, "The Cheng-Minkowycz problem for natural convective boundary layer flow in a porous medium saturated by a nanofluid: a revised model," International Journal of Heat and Mass Transfer, vol. 65, pp. 682-685, 2013.

[13] P. Rana, R. Bhargava, and O. A. Bégb, "Numerical solution for mixed convection boundary layerflow of ananofluid along an inclined plate embedded in a porous medium," Computers and Mathematics with Applications, vol. 64, no. 9, pp. 2816-2832, 2012.

[14] A. M. Rashad, A. J. Chamkha, and M. Modather, "Mixed convection boundary-layer flow past a horizontal circular cylinder embedded in a porous medium filled with a nanofluid under convective boundary condition," Computers \& Fluids, vol. 86, pp. 380-388, 2013.

[15] M. Sheikholeslami and D. D. Ganji, "Nanofluid flow and heat transfer between parallel plates considering Brownian motion 
using DTM," Computer Methods in Applied Mechanics and Engineering, vol. 283, pp. 651-663, 2015.

[16] N. V. Ganesh, B. Ganga, and A. K. Abdul Hakeem, "Lie symmetry group analysis of magnetic field effects on free convective flow of a nanofluid over a semi-infinite stretching sheet," Journal of the Egyptian Mathematical Society, vol. 22, no. 2, pp. 304-310, 2014.

[17] Y. Lin, L. Zheng, and G. Chen, "Unsteady flow and heat transfer of pseudo-plastic nanoliquid in a finite thin film on a stretching surface with variable thermal conductivity and viscous dissipation," Powder Technology, vol. 274, pp. 324-332, 2015.

[18] M. Sheikholeslami, M. M. Rashidi, and D. D. Ganji, "Numerical investigation of magnetic nanofluid forced convective heat transfer in existence of variable magnetic field using two phase model," Journal of Molecular Liquids, vol. 212, pp. 117-126, 2015.

[19] T. Hayat, M. Waqas, S. A. Shehzad, and A. Alsaedi, "Mixed convection flow of a Burgers nanofluid in the presence of stratifications and heat generation/absorption," The European Physical Journal Plus, vol. 131, no. 8, p. 253, 2016.

[20] F. M. Abbasi, T. Hayat, S. A. Shehzad, F. Alsaadi, and N. Altoaibi, "Hydromagnetic peristaltic transport of copper-water nanofluid with temperature-dependent effective viscosity," Particuology, vol. 27, pp. 133-140, 2016.

[21] M. Sheikholeslami, D. D. Ganji, and M. M. Rashidi, "Magnetic field effect on unsteady nanofluid flow and heat transfer using Buongiorno model," Journal of Magnetism and Magnetic Materials, vol. 416, pp. 164-173, 2016.

[22] O. Pourmehran, M. Rahimi-Gorji, and D. D. Ganji, "Heat transfer and flow analysis of nanofluid flow induced by a stretching sheet in the presence of an external magnetic field," Journal of the Taiwan Institute of Chemical Engineers, vol. 65, pp. 162-171, 2016.

[23] M. Rahimi-Gorji, O. Pourmehran, M. Hatami, and D. D. Ganji, "Statistical optimization of microchannel heat sink (MCHS) geometry cooled by different nanofluids using RSM analysis," European Physical Journal Plus, vol. 130, no. 2, article 22, pp. 121, 2015.

[24] W. Ibrahim and B. Shankar, "MHD boundary layer flow and heat transfer of a nanofluid past a permeable stretching sheet with velocity, thermal and solutal slip boundary conditions," Computers \& Fluids, vol. 75, pp. 1-10, 2013.

[25] A. Malvandi and D. D. Ganji, "Brownian motion and thermophoresis effects on slip flow of alumina/water nanofluid inside a circular microchannel in the presence of a magnetic field," International Journal of Thermal Sciences, vol. 84, pp. 196206, 2014.

[26] R. Ul Haq, S. Nadeem, Z. H. Khan, and N. S. Akbar, “Thermal radiation and slip effects on MHD stagnation point flow of nanofluid over a stretching sheet," Physica E, vol. 65, pp. 17-23, 2015.

[27] M. Govindaraju, S. Saranya, A. A. Hakeem, R. Jayaprakash, and B. Ganga, "Analysis of slip MHD nanofluid flow on entropy generation in a stretching sheet," Procedia Engineering, vol. 127, pp. 501-507, 2015.

[28] M. J. Uddin, M. N. Kabir, and O. A. Bég, "Computational investigation of Stefan blowing and multiple-slip effects on buoyancy-driven bioconvection nanofluid flow with microorganisms," International Journal of Heat and Mass Transfer, vol. 95, pp. 116-130, 2016.
[29] K.-L. Hsiao, "Stagnation electrical MHD nanofluid mixed convection with slip boundary on a stretching sheet," Applied Thermal Engineering, vol. 98, pp. 850-861, 2016.

[30] P. K. Kameswaran, M. Narayana, P. Sibanda, and P. V. S. N. Murthy, "Hydromagnetic nanofluid flow due to a stretching or shrinking sheet with viscous dissipation and chemical reaction effects," International Journal of Heat and Mass Transfer, vol. 55, no. 25-26, pp. 7587-7595, 2012.

[31] M. H. Matin and I. Pop, "Forced convection heat and mass transfer flow of a nanofluid through a porous channel with a first order chemical reaction on the wall," International Communications in Heat and Mass Transfer, vol. 46, pp. 134-141, 2013.

[32] D. Pal and G. Mandal, "Influence of thermal radiation on mixed convection heat and mass transfer stagnation-point flow in nanofluids over stretching/shrinking sheet in a porous medium with chemical reaction," Nuclear Engineering and Design, vol. 273, pp. 644-652, 2014.

[33] C. Zhang, L. Zheng, X. Zhang, and G. Chen, "MHD flow and radiation heat transfer of nanofluids in porous media with variable surface heat flux and chemical reaction," Applied Mathematical Modelling, vol. 39, no. 1, pp. 165-181, 2015.

[34] H. M. Elshehabey and S. E. Ahmed, "MHD mixed convection in a lid-driven cavity filled by a nanofluid with sinusoidal temperature distribution on the both vertical walls using Buongiorno's nanofluid model," International Journal of Heat and Mass Transfer, vol. 88, pp. 181-202, 2015. 

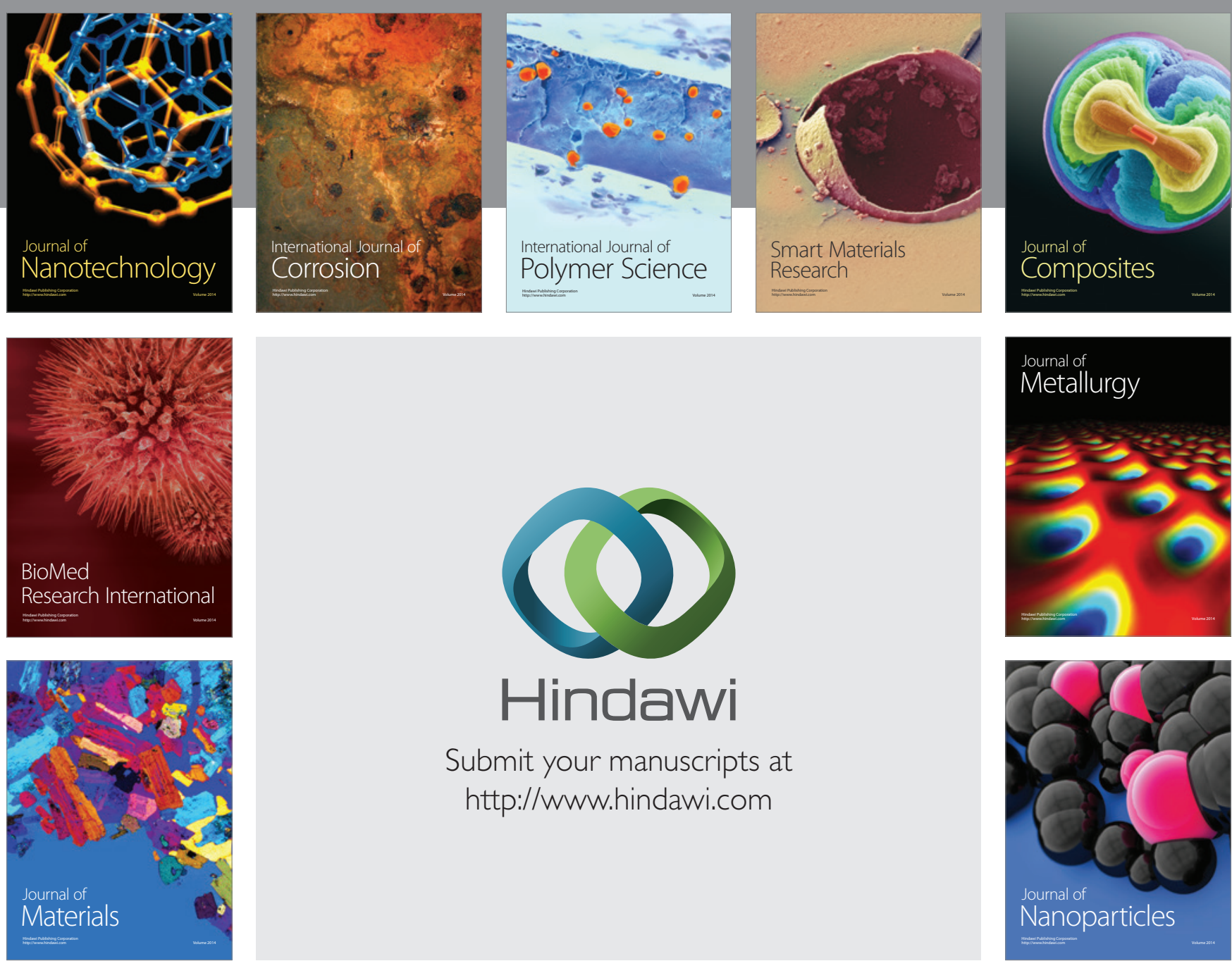

\section{Hindawi}

Submit your manuscripts at

http://www.hindawi.com

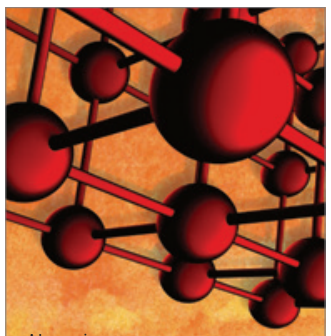

Materials Science and Engineering
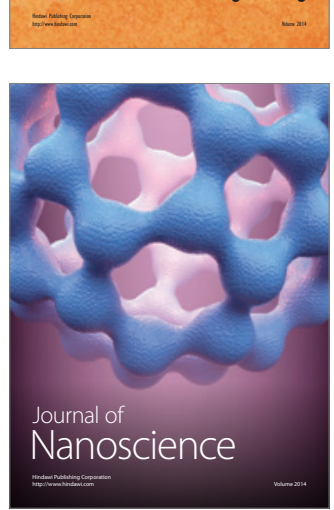
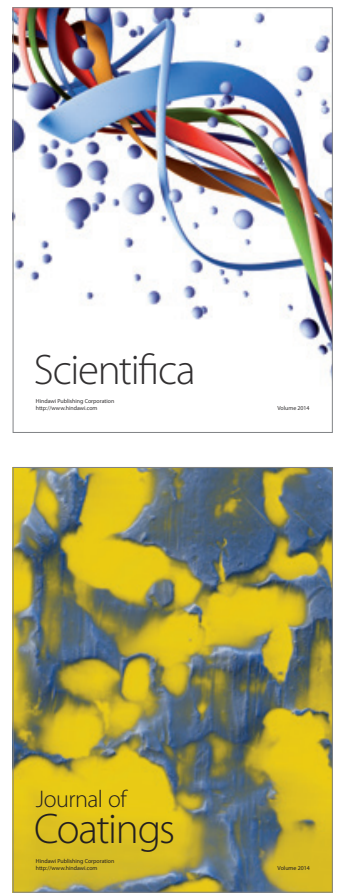
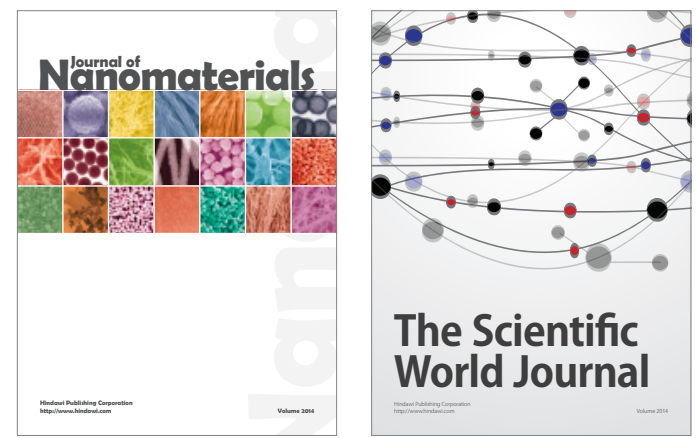

The Scientific World Journal
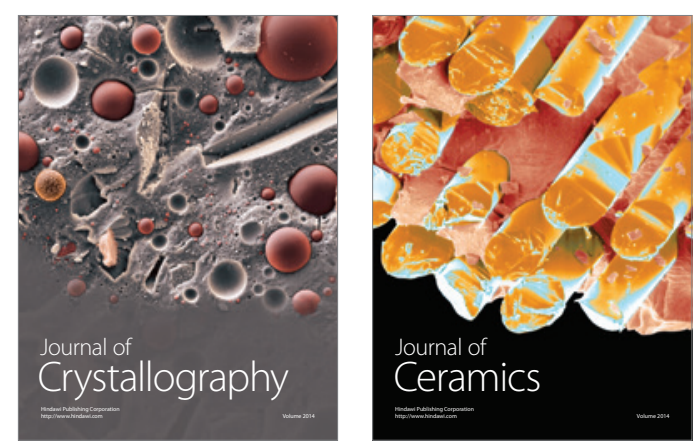
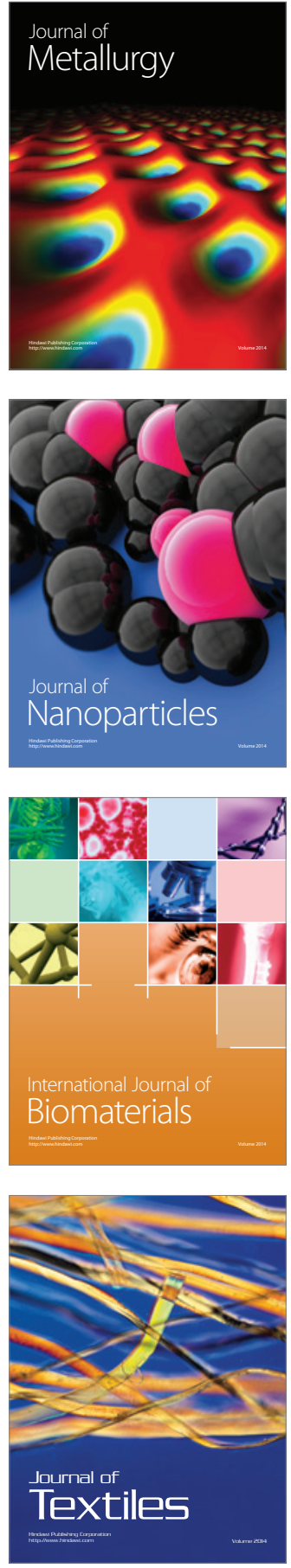